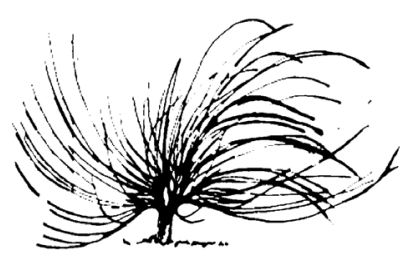

\title{
La universidad constituyente: una vía hacia la transdisciplinariedad
}

\author{
José Acosta ${ }^{1}$ \\ Universidad Politécnica Territorial del Oeste de Sucre Clodosbaldo Russián \\ Venezuela \\ acostajjg@gmail.com
}

\begin{abstract}
Resumen
El propósito del presente ensayo consiste en abordar, desde una mirada crítica, la posibilidad de encauzar la transdisciplinariedad desde la universidad constituyente, como perspectiva para superar la universidad constituida, esta última es entendida como aquella que se sustenta en la lógica disciplinar para reproducir, validar y tornar hegemónico el conocimiento producido desde la racionalidad científica. La academia, desde la mirada constituyente, coadyuvaría con la generación de otros campos del saber a partir del diálogo entre disciplinas. El trabajo investigativo, por tanto, se asumió desde la hermenéutica que, como ejercicio crítico y reflexivo, favoreció el abordaje de categorías como educación, conocimiento, humanismo, las cuales dejan entrever un cambio a lo interno de la configuración del saber que, desde la transdisciplinariedad, pueda construir otros espacios pedagógicos para dinamizar la interrelación de las funciones desempeñadas por todos sus protagonistas, lo que fractura así el amarre con la lógica disciplinaria. Dicha ruptura, para la universidad constituyente, ofrecería la oportunidad de imbricar el conocimiento científico con los saberes legos,
\end{abstract}

\section{(C) $(1 \Theta \Theta$}

Recibido: 11 de julio de 2019. Aprobado: 29 de abril de 2020.

http://dx.doi.org/10.15359/rep.15-2.4

1 Licenciado en Física. Magíster en Educación Mención Enseñanza de la Física. Doctor en Educación. Docente Asociado. Vicerrector Académico (E). Universidad Politécnica Territorial del Oeste de Sucre Clodosbaldo Russián (UPTOSCR). Cumaná, Sucre, Venezuela. 
populares, cotidianos, que circulan en el ámbito social y sientan las bases para un nuevo tipo de humanismo.

Palabras clave: diálogo de saberes, educación universitaria, transdisciplinariedad

\begin{abstract}
The purpose of this paper is to address, from a critical perspective, the possibility of channeling transdisciplinarity from the constituting university, as a perspective to overcome the constituted university; the latter is understood as that which is based on the disciplinary logic to reproduce, validate, and make the knowledge produced from scientific rationality hegemonic. The university, from the constituting perspective, would contribute to the generation of other fields of knowledge as a result of the dialogue between disciplines. The investigative work, therefore, was assumed from hermeneutics, which, as a critical and reflective exercise, favored the approach to categories, such as education, knowledge, humanism, which allow us to glimpse a change within the configuration of knowledge that, from transdisciplinarity, can build other pedagogical spaces to dynamize the interrelation of the functions performed by all its protagonists, thus fracturing the mooring with disciplinary logic. This rupture, for the constituting university, would offer the opportunity to imbricate scientific knowledge with lay wisdow, popular, everyday knowledge that circulates in the social field laying the foundations for a new type of humanism.
\end{abstract}

Keywords: Dialogue of knowledge, university education, transdisciplinarity

\title{
Introducción
}

T a universidad se ha transfigurado, en el tiempo presente, en una organización compleja, lo cual está relacionado con la variedad de Lunciones que desempeña, a la heterogeneidad de los colectivos que la conforman y a la escabrosa red de relaciones entre sus integrantes. Un arqueo bibliográfico sobre ella nos encauza por los intrincados senderos de los vínculos, prácticas, códigos y símbolos que conviven, en muchos casos incompatiblemente, a lo interno de la institución educativa. El transitar por estas sendas deja entrever una diversidad de identidades y racionalidades que brotan de la división del trabajo, la lógica disciplinar, la 
fragmentación del conocimiento, los intereses divergentes y congruentes de los sujetos. El conflicto por el estatus y el reconocimiento proyectan, parcialmente, la sociedad en su conjunto.

Adicionalmente, vale decir que "la gran expansión del conocimiento científico en tantas disciplinas ha alentado currículos mono-disciplinarias ... Los efectos de estas disyunciones pueden ser insidiosas y minar una visión común de la naturaleza y el propósito de la empresa académica" (Mitchell, 2000, según se cita en Salazar, 2003, p. 8). Vale resaltar que el conocimiento científico:

fue a lo largo del siglo XX un conocimiento predominantemente disciplinar, cuya autonomía impone un proceso de producción relativamente descontextualizado con relación a las necesidades del mundo cotidiano de las sociedades ... es homogéneo y organizativamente jerárquico en la medida en que los agentes que participan en su producción comparten los mismos objetivos de producción de conocimiento, tienen la misma formación, la misma cultura científica y lo hacen según jerarquías organizacionales bien definidas. (De Sousa, 2008, p. 25)

Durante casi todo el siglo XX, con base en lo anterior, la universidad, desde la mirada constituida, se instituyó como un espacio aislado del contexto social en la que la idea reduccionista, fragmentada y antropocéntrica de la realidad se refleja, por un lado, en la hegemonía del conocimiento científico y, por otro, en la estructuración del currículo. Apoyadas en estos referentes, "sobresalen las prácticas pedagógicas que promueven la transmisión acrítica de los saberes validados con énfasis en la acumulación y transmisión de información, el dominio y aplicación de contenidos y técnicas y la simplificación-fragmentación del conocimiento, entre otras cosas" (Becerra, 1996, p. 49).

En contraposición a la idea precedente, desde finales del siglo pasado e inicios del presente, han surgido nuevas perspectivas que aportan otras miradas que responden a los cambios epistemológicos, filosóficos y metodológicos tanto de las ciencias humanistas como de las naturales. La universidad debe asumir sus necesidades y las de la sociedad, para ello, por una parte, ha de aceptar esta nueva realidad y, por otra, emprender los procesos formativos e investigativos que marquen distancia con la hegemonía de la lógica disciplinaria, esta última es "esencialmente 
un modelo cognitivo cuya eficacia consiste en la enorme cantidad de presupuestos epistemológicos -subrepticios- con los que trabaja ... lleva aparejada una alta propensión a la super-especialización: el conocer cada vez más sobre cada vez menos" (Lanz, 2010, p. 201).

Ante este horizonte, concebimos la otredad pedagógica que denominamos universidad constituyente. Lo constituyente sería la comprensión crítica de las formas socio-culturales donde lo vivido simbolice la expresión de un proceso formativo e investigativo abierto, que represente la separación con aquellas cosmovisiones simbólicas y prescriptivas en los espacios educativos. Pensar una universidad constituyente, que permita superar los obstáculos que erija este siglo, ha de irrigar la transversalización entre el conocimiento científico con los saberes humanistas, populares, cotidianos, en otras palabras, con la cultura que circula en la sociedad. Esta transversalización ha de promover la imbricación activa de saberes bajo el argumento de que todos ellos, incluido el conocimiento científico, se pueden enriquecer en esa convivencia, la cual determinaría, a su vez, el quiebre con la lógica disciplinaria.

Desde este panorama, se manifiestan las siguientes interrogantes: $¿$ nos inclinaremos a favor de una universidad constituyente o permaneceremos anclados a la universidad constituida?, ¿la transdisciplinariedad podrá sustentar la universidad constituyente para superar la hegemonía disciplinaria? Responder estas interrogantes no es nada sencillo, pero tengamos presente que el acaecimiento de una nueva sociedad no será posible sin una universidad que asuma todas las dimensiones del ser humano.

Razón por la cual, el objetivo de este trabajo consistió en concebir la universidad constituyente desde la transdisciplinariedad. Lo cual permitiría una visión compleja de la realidad al tiempo que sirva de ámbito para incorporar a los sujetos al mundo profesional sin desligarlo de su contexto social, en oposición con la visión compartimentada y aislada de las diferentes disciplinas insertas en la universidad constituida.

Con base en lo anterior, el presente trabajo se abordó desde la perspectiva hermenéutica, lo que permitió desarrollar un imaginario conceptual para dialogar con la imaginación cultural y el simbolismo que encierra el encuentro con los seres humanos. Por eso, el concepto de universidad constituyente es un entretejido de ideas de carácter crítico-reflexivo. El ejercicio hermenéutico, por tanto, favoreció un viaje a través del ámbito textual de diversos autores, al comprender que cada escrito es un territorio de posibilidades para la actividad reflexiva, en 
otras palabras, contribuyó a "comprender el sentido de lo dicho por el autor, lo que no significa tal como el autor lo ha entendido, sino un ir más allá de la comprensión del autor" (Gadamer, 1998, según se cita en Castro, 1998, p. 72).

Al considerar la hermenéutica como recurso para ennoblecer el pensamiento, se genera una toma de conciencia respecto al valor de lo socio-educativo como espacio múltiple, lo cual ensancha el sentido de la universidad más allá de los sistemas tradicionales, al fortalecer una base cultural donde emerge la "pluralidad en las relaciones del hombre con el mundo, en la medida en que responde a la amplia variedad de sus desafíos, en que no se agota en un solo tipo ya establecido de respuesta" (Freire, 1980, p. 28).

La concepción de la universidad constituyente, esbozada en este escrito, se nutre, de manera conceptual de diversos aportes, incorporándolos dialécticamente a través del ejercicio hermenéutico, haciéndose ella misma hermenéutica de lo educativo, interpretando el caudal de vida del ser en sociedad generando cultura.

Por otra parte, al plantear la concepción del objeto en términos de la transdisciplinariedad, se escudriña el diálogo con diferentes autores para replantear el propio objeto a la altura de los conceptos que lo integran y suponer el alcance que pudieran tener como propuesta. En consecuencia, comprender que el corolario de dichos diálogos obra "en nuestro interior produciendo también, y necesariamente, transitividad de la conciencia, cuyos resultados son niveles progresivos de reflexividad" (Cubillán, 2008, p. 25), rasgos necesarios en la producción de la teoría para la formulación del entramado conceptual aquí planteado.

El ejercicio hermenéutico, por tanto, fue una invitación para "crear los nuevos universos umbilicalmente unidos al universo de su vida práctica, del imaginario y de las ideas" (Morin, 1992, p. 77). Tal invitación, permitió liberar el pensamiento de las restricciones impuestas en la universidad constituida, e ir más allá, hacia conglomerados socio-culturales más complejos: la universidad constituyente.

\section{La universidad constituida y su concepción de disciplina}

La disciplinariedad tiene un correlato en la creación de las universidades en el siglo XIX, razón por la cual, las disciplinas tienen un devenir histórico que se inserta en la historia de la sociedad. Por ello, la disciplinariedad posee una dimensión epistemológica y paradigmática abocada al 
entendimiento de los modelos de estructuración del conocimiento disciplinar y sus procesos de clausura y apertura (Morin, 2002). Las maneras de organización del conocimiento en la educación universitaria, sus modos y desarrollos, según Lanz y Fergusson (2005), están más relacionados con formas de organización que con órdenes establecidos. Por lo tanto, la disposición de los colectivos académicos, como comunidades de sentido, se constituye en fundamento desde el cual se conforman las miradas pedagógicas, el ordenamiento de las ciencias en núcleos epistemológicos y la distribución de los procesos de formación e investigación.

La noción de disciplina, en esta trama, puede ser entendida como una categoría organizadora dentro del conocimiento científico, instituyendo así la especialización. Según Morin (1993):

La especialización abs-trae, es decir, extrae un objeto de un campo dado ... lo inserta en un sector conceptual abstracto que es el de la disciplina compartimentada, cuyas fronteras rompen arbitrariamente la sistematicidad (la relación de una parte con el todo) y la multidimensionalidad de los fenómenos ... privilegiando, por un lado, todo lo que es calculable y formalizable e ignorando, por el otro, el contexto necesario para la inteligibilidad de sus objetos. (p. 189)

En este sentido, por más que se inserta en un contexto científico más amplio una disciplina tiende a la autonomía por medio de la delimitación de sus fronteras en el nivel técnico, metodológico y epistemológico (Morin, 2002). Las disciplinas, cabe resaltar, tomaron forma al darse la separación entre ciencia y filosofía por la gran diversidad de objetos de estudio que florecieron luego de la llamada revolución científica, cuando fueron superados muchos de los límites impuestos por los dogmatismos religiosos y culturales. Así, cada disciplina se enclaustra en una arista según su objeto de estudio específico.

La demarcación de una parte del universo como objeto de interés específico de una disciplina fue un proceso aparentemente inevitable en el desarrollo humano. Es por ello que "la disciplina es una categoría organizadora dentro del conocimiento científico; instituye en éste la división del trabajo y responde a la diversidad de dominios que recubren las ciencias" (Morin, 2002, p. 115). Podría decirse, entonces, que la ciencia moderna se desarrolló gracias al establecimiento de las disciplinas. 
De esta manera, la división de la realidad en diferentes fracciones de análisis surgió como una opción sugestiva para que los sujetos pudiesen acceder a la verdad fundamental del mundo, y de sí mismos. La separación progresiva de determinados campos de conocimiento, provocado por la ciencia moderna, derivó en hiperespecialización definida como aquella que "se encierra en ella misma sin permitir su integración en una problemática global o en una concepción de conjunto del objeto del que solo considera un aspecto o una parte" (Morin, 2002, p. 13). Los objetos de estudio, con el transcurrir del tiempo, se fueron haciendo más diversos y específicos, en consecuencia, había mucho por investigar sobre un objeto en particular.

El conocimiento científico hiperespecializado se fue incrementando y, paralelamente, la posibilidad de acceder a una visión global de la realidad se tornó cada vez más efímera. Los actores universitarios se convirtieron en especialistas inmersos en el micromundo de su disciplina. Así, dichos sujetos conocen "cada vez más sobre menos hasta que saben todo sobre nada, ven el árbol y se pierden en el bosque, confunden el todo con las partes y creen saber a dónde van y no lo saben" (Morles, 2004, p. 56), en otras palabras, pierden la visión de contextualizar, de "situar una información o un saber en su contexto natural ... para introducir los conocimientos en un conjunto más o menos organizado" (Morin, 1997, p. 1).

A mediados del siglo XX, cuando los niveles de especialización se tornan demasiado agudos, comienzan a brotar objetos de estudio difíciles de abordar desde una sola disciplina. Entre ellos se tienen: el deterioro ambiental, la crisis energética y la escasez de alimentos. Se hacía necesario, dada la magnitud de los problemas, sumar esfuerzos para poder dar una visión de conjunto sobre estas problemáticas.

El sumar esfuerzos, cabe destacar, para afrontar un problema de manera integral y compleja acarrea necesariamente la instauración de un diálogo entre disciplinas. Por lo que es necesario tomar conciencia de que:

A partir de las disciplinas ... es posible reconocer la unidad y complejidad humana reuniendo y organizando conocimientos dispersos en las ciencias de la naturaleza, en las ciencias humanas, la literatura, la filosofía y mostrar la unión indisoluble entre la unidad y la diversidad de todo lo que es humano. (Morin, 1999, p. 15) 
Se requiere, en consecuencia, la construcción de puentes que permitan la comunicación entre las disciplinas para el intercambio e integración de teorías y metodologías, lo que provoca transformaciones en estas. Debemos tener en cuenta que el diálogo entre disciplinas posibilitará la influencia de unas sobre las otras, también nivelaría el camino para la germinación de nuevas categorías que se desarrollarían desde otros lenguajes, métodos y objetos de estudio diferentes a los que les dieron origen. Cabe resaltar que la lógica disciplinaria, presente en la universidad constituida, no ha generado una total y absoluta inercia en la misma ya que desde ella se han abierto caminos para:

superar la desigualdad social, la fragmentación del saber ... para promover la multiculturalidad a través de un currículo integrado y humanista que enfatice sobre los valores propios del saber histórico, las costumbres regionales y nacionales, los recursos naturales y el patrimonio cultural. (Acosta, 2017, p. 57)

Son estos aspectos, propiciados desde la universidad constituida, los que hacen pensar la universidad como "un espacio público de interconocimiento" (De Sousa, 2008, p. 95), lo que deja entrever que estamos ante la emergencia de nuevas perspectivas, entre ellas la transdisciplinariedad, que coadyuvan con la imbricación disciplinaria en el ámbito del saber de las ciencias, dada la insuficiencia de las explicaciones del conocimiento científico, el cual rechaza muchas miradas que contribuyen a comprender la realidad humana en toda su complejidad.

\section{Sobre la perspectiva transdisciplinaria}

La transdisciplinariedad, desde el punto de vista educativo, podría entenderse como una bifurcación al interior de la configuración del conocimiento. Lo que permitiría incorporar, por un lado, saberes desestimados por la lógica disciplinaria y, por otro, identidades, maneras de concebir el conocimiento, diversidad cultural, experiencias y vivencias. Es por ello que:

a la transdisciplinariedad concierne lo que está, a la vez, entre las disciplinas, a través de las diferentes disciplinas y más allá de toda disciplina. Su finalidad es la comprensión del mundo 
presente, y uno de sus imperativos es la unidad del conocimiento. (Nicolescu, 1996, p. 37)

Así, el prefijo trans no denota pérdida de identidad, sino más bien relación, una condición inmanente al diálogo entre disciplinas, estrechamente conectadas y dependientes entre sí. Los nuevos horizontes que aporta la transdisciplinariedad, en el ámbito del conocimiento, han de trasladarse a la educación universitaria por cuanto esta, en su sentido más amplio, constituye uno de los recursos más valiosos para emprender las transformaciones necesarias acordes con los nuevos tiempos. Contribuye así con la reforma del pensamiento para afrontar la complejidad creciente, la rapidez de los cambios y lo imprevisible de nuestro mundo.

Una de las transformaciones, por generar desde la reforma del pensamiento, tiene que ver con la fractura de la racionalidad científica que en el ámbito universitario:

Se ha institucionalizado, imponiéndose como la forma de producción del conocimiento. Es decir, en nuestros centros de educación superior encontramos ... unidades específicas de investigación donde «legítimamente» se produce «conocimiento científico» y ellos dan pautas, los criterios, el modelo para producción, incluso, determina, con autoridad, en gran parte, lo que ha de producir. (Becerra, 1997, p. 64)

Hay que cambiar, por tanto, la manera de razonar arraigada en la universidad constituida, su memorismo normativo, su reproducción simple. La sociedad del tiempo presente requiere una racionalidad diferente, enlazada con las iniciativas, la cooperación, el sentido de la responsabilidad, la ética, la capacidad de relacionar fenómenos; que asume, en todo momento, los brotes emergentes de lo nuevo.

Para alcanzar tal propósito, se debe replantear, entre otros, la organización del conocimiento, reformular políticas y programas educativos, al perdurar las expectativas en las generaciones futuras, las cuales han de estar dispuestas para hacer frente a los desafíos que se manifiestan tanto en la cultura científica como en la socio-humanista.

En este sentido, el camino de la educación universitaria se entrelaza con la compleja trama del tejido social, porque es el resultado de fuerzas vitales que conllevan al desarrollo, así como al estímulo 
intelectual y la voluntad de cambio. De manera antagónica a la concepción que se tiene de universidad constituida, como retén de la tradición, la nueva universidad ha de emerger con el signo de la transformación, lo cual las comprometerá con las grandes innovaciones de nuestro tiempo y los requerimientos de la sociedad.

Debido al alcance y ritmo de las transformaciones, vale decir, la sociedad tiende cada vez más a basarse en el conocimiento, por lo que la formación y la investigación son parte esencial del desarrollo cultural, social, económico, educativo y político de las regiones. Por tanto, tendrá que hacer frente a excepcionales obstáculos: ha de emprender la renovación más esencial, de forma tal, que la sociedad contemporánea pueda apropiarse de los argumentos necesarios para asumir rasgos de sensibilidad que le den otro sentido a la vida.

Para alcanzar tales metas, la nueva universidad deberá desarrollar una formación a la cual se le pueda "demandar pruebas de validación que estén referidas a su consistencia teórica, a su coherencia lógica y pertinencia social ... para responder al desarrollo endógeno" (Lanz, 2007 , p. 1) y una investigación que "no se conciba ... como un proceso lineal o metódico único, más bien incorpora la complejidad y la transdisciplinariedad para el logro de los procesos reflexivos de la ciencia" (González, s. f. , p. 114). Estos aspectos permitirán reconocer que "el pensamiento transdisciplinario y el pensamiento complejo hablan desde la misma tribuna, son en verdad una unidad ... La complejidad es del conocimiento y los procesos reales; la transdisciplinariedad de los nuevos modos de producción de conocimiento" (Lanz, 2010, p. 206).

Lo anterior lleva hacia la reflexión de la educación universitaria, desde la perspectiva transdisciplinaria, como dimensión clave para la nueva universidad. Como destaca Nicolescu (1996):

el enfoque transdisciplinario puede hacer una contribución importante para el advenimiento de un nuevo tipo de educación ... Para ello, es indispensable el espíritu científico, como una de las mayores adquisiciones de la aventura humana; igualmente, es importante la iniciación precoz en las ciencias, lo cual permite el acceso, desde el principio de la vida humana, a la inagotable riqueza del espíritu científico, basado en el cuestionamiento, en el rechazo a cualquier respuesta prefabricada y a cualquier certeza que contradiga los hechos. Ahora bien, el espíritu científico no 
significa en absoluto el aumento desenfrenado de la enseñanza de materias científicas ... No es, entonces, la asimilación de una masa de conocimientos científicos lo que permite el acceso al espíritu científico, sino la calidad de lo que se enseña; y «calidad» quiere decir hacer penetrar al niño, al adolescente o al adulto en el corazón mismo del proceso científico, que es el cuestionamiento permanente en relación con la resistencia a los hechos, las imágenes, las representaciones y las formalizaciones. (p. 54)

La transdisciplinariedad, con base en lo anterior, lleva a la trasgresión de la lógica disciplinaria lo que abriría las puertas para que el espíritu científico asuma la diversidad y el "reconocimiento de la existencia de una pluralidad de conocimientos más allá del conocimiento científico" (De Sousa, 2010, p. 48). Así, los sujetos podrán salir de los cánones establecidos por la lógica disciplinaria e impregnar, con esta visión, el quehacer formativo e investigativo coadyuvando al desarrollo de una universidad más integral y versátil, dispuesta a dar soluciones a problemas de la realidad.

La admisión de la transdisciplinariedad, en la nueva universidad, se podrá avizorar a través de la ejecución de planes y programas que cubran las expectativas de los actores universitarios, en cuanto a la formación pertinente de su espíritu científico. Para ello, será necesario aventajar la concepción fragmentaria del currículo a través de, entre otras, la vinculación de la investigación:

con las políticas sociales, en proyectos de cierta complejidad que permitan la participación de todos los actores involucrados. Proyectos de este tipo, de una trascendencia cónsona a la importancia social de los problemas a abordar, deben ser organizados en los ámbitos de la política educativa, de salud, de cultura, entre otros. Solo una vinculación con los organismos ejecutores de dichas políticas, así como con los sectores sociales involucrados, podrá garantizar la transversalización y aplicación de los saberes. (Acosta, 2018, p. 13)

Es imperativo romper con la fragmentación curricular, que aísla a la ciencia de la cultura y el humanismo, por medio de la investigación coadyuvaría para alcanzar "una educación que se dirige a la totalidad 
abierta del ser humano y no a uno solo de sus componentes" (Nicolescu, 1996, p. 96) y así formar un espíritu más humano, sensible y ético. Razón por la cual:

Es necesaria una revolución en el pensamiento, en la elaboración de nuestras construcciones mentales y en su representación. En pos de esas finalidades, la conjunción de las nuevas tecnologías con los métodos transdisciplinarios es la que puede ofrecer una inteligencia estratégica y a la vez estrategias inteligentes para que las "mundializaciones" en marcha desemboquen en una verdadera y nueva civilización, y no en una nueva barbarie, en un tiempo en el que prosiga la evolución del ser humano, el más intenso crecimiento de su humanidad. (Vilar, 1997, p. 69)

Asumir integralmente la mirada pedagógica y tecnológica en la educación universitaria, ofrece una oportunidad para encaminar la transdisciplinariedad al desarrollar habilidades y disposiciones generales, que permitirán al sujeto desenvolverse pertinentemente tanto en los contextos sociales como profesionales, y aportar respuestas a las distintas situaciones que enfrente. Como destaca Nicolescu (1996):

La adquisición de un oficio pasa necesariamente por una especialización ... Sin embargo, en nuestro mundo en ebullición, donde el sismo informático anuncia otros sismos futuros, dedicarse toda la vida a un único y mismo oficio sería peligroso, puesto que podría conducir al desempleo, a la exclusión y al sufrimiento que desintegra al ser. La especialización excesiva y precoz tiende a abolirse de un mundo en constante cambio. Si queremos conciliar exigencia competitiva y preocupación por igualdad de oportunidades para todos los seres humanos, todo oficio en el futuro tendrá que ser un oficio que se pueda tejer, un oficio que esté religado al interior del ser humano y a los hilos que lo religan con otros oficios. Desde luego, no se trata de adquirir varios oficios a la vez, sino de construir un núcleo flexible que permita rápidamente el acceso a otro oficio. (p. 94)

De allí que se asuma la transdisciplinariedad como perspectiva mediante la cual se configuran redes de formación e investigación, que 
manifiesten la integración entre la teoría y la práctica, entre el método y el contenido, para transformar el pensamiento de los actores educativos y sociales al generar otros modos de actuación en el mundo. Una vía para alcanzar este horizonte sería a través de una nueva universidad que, sustentada en la transdisciplinariedad, aperture escenarios inéditos para:

volverse un lugar de aprendizaje de la actitud transcultural, transreligiosa, transpolítica y transnacional, del diálogo entre el arte y la ciencia, eje de la reunificación entre la cultura científica y la cultura artística. La Universidad renovada será el hogar de un nuevo humanismo. (Nicolescu, 1996, p. 98)

\section{Hacia la concepción de la universidad constituyente como posibili- dad de encauzar la transdisciplinariedad}

La transdisciplinariedad deja entrever que la cultura científica y la socio-humanista no pueden ser conocidas fuera de sus relaciones con el ser humano, por lo que a dicha perspectiva concierne la generación de un saber cada vez más complejo de la realidad. La educación, por tanto, ha de fundarse en la reconsideración del papel de la intuición, la imaginación, lo sensibilidad, lo cotidiano, entre otros. Las universidades, muy particularmente, han de reestructurarse, renovarse, para abordar la realidad en la que están inmersas.

Con base en lo anterior, la universidad constituyente, como campo roturado para la transdisciplinariedad, entrañaría la generación de espacios epistemológicos para la expresión de la subjetividad, para la generación de otra lógica y de nuevas categorías que estimulen un auténtico diálogo entre las diversas disciplinas. Por esto, en lugar de una simple transferencia de conocimientos desde una disciplina a otra, la universidad constituyente ha de tomar en cuenta la circulación de saberes que fluyen entre varias ramas del conocimiento, así como de los ámbitos socioculturales de los que brotan.

La transdisciplinariedad, como perspectiva epistemológica, coadyuvaría para que la universidad constituyente no sea concebida como ámbito para la transmisión y validación del conocimiento científico. Dicha universidad, por el contrario, ha de representar el rastreo de contextos en los que las disciplinas puedan relacionarse, dialógica y dialécticamente, para exteriorizar las complejas y diversas relaciones que asisten 
al diálogo en la construcción del saber en los espacios de constitución de subjetividades. En lo educativo, vale decir, se expresan los distintos sentidos subjetivos y estos representan las múltiples contribuciones individuales que en la discusión se tornarían colectivos; de allí brotarán miradas que representen intentos para abordar la diversidad cultural.

Desde esta última, en la que se transversalizan distintos saberes y experiencias para dinamizar la transdisciplinariedad, la universidad constituyente promovería la germinación de un pensamiento que permita canjear los:

imperativos lógicos de las reglas metodológicas por una recuperación del movimiento de la razón por medio de la dialéctica: rescatar el problema lógico en el plano de un razonamiento no restringido a lo formal sino abierto a relaciones más amplias e inclusivas de la realidad. (Zemelman, 1992, p. 88)

La universidad constituyente, según lo anterior, no daría oportunidad al pensamiento enmarcado en los límites disciplinarios. Por esto, la misma se constituiría en horizonte para pensar lo no pensado, en ámbito de confluencia y trascendencia de sí mismo como forma de transversalización de los saberes, lo que conlleva una permanente transformación que desplomaría los muros que aíslan las disciplinas.

Emerge, entonces, la siguiente interrogante: ¿cómo definir la visión transdisciplinaria de una realidad desde la universidad constituyente? Nos aventuramos a definirla como la aprehensión de esa realidad en un entorno más amplio ofrecido por las diversas disciplinas involucradas en la educación universitaria, las cuales se imbrican consolidando el sentido de la totalidad.

La pertinencia de este contexto epistemológico transdisciplinario es que la educación universitaria se fundamentaría en la posibilidad de generar, por medio del diálogo entre disciplinas, otros escenarios para comprender y explicar la realidad. Lo que se pretende es viabilizar, desde la universidad constituyente, intercambios dialéctico compresivos e intersubjetivos que atraviesen y trasciendan las disciplinas. Así, desde una visión compleja, dicha universidad supone otra lectura de categorías interconectadas sustentada en el pliegue, repliegue y despliegue de una relación dialéctica y dialógica que implica, como acción formativa, la reincorporación del ser humano en el acto del conocimiento y el 
saber. Cabe resaltar, que una vía para hacer dialogar las disciplinas en la universidad constituyente sería a través de la inserción de ejes de formación los cuales podrían ser concebidos:

como aquellos que implican concientización, reencuentro o interencuentro con las bases ontológicas y epistemológicas que imbrican las disciplinas con la cultural que circula en la sociedad. Estos ejes dinamizarán la emergencia de valores, prácticas, vivencias, saberes y experiencias sustentadas en la imaginación y la sensibilidad, conformando de esta manera el proceso de formación integral, igualmente, acogerán valores humanos, culturales e históricos, que permitan abordar las problemáticas más apremiantes de la sociedad ... Desde esta perspectiva, se podrá vitalizar una formación que no esté centrada exclusivamente en la especialización, sino que conllevará hacia una visión más humanista que trascienda a la sociedad e implique una conciencia para la vida. (Acosta, 2016, p. 54)

Lo transdisciplinario, visto como ruta de comunicación y encuentro, supone que lo que está entre, a través y más allá de las disciplinas compone un entorno de posibilidades para las múltiples formas de crear, cocrear y recrear el conocimiento porque los imbrica a todos y supone su transformación, al rotular su propio espacio. Aspecto que contribuiría a sentar las bases de la universidad constituyente para avivar principios como la libertad, el diálogo, el respeto y la diversidad. Lo que sobresale, así mismo, por el rastreo de otra lógica de sentido y por atisbar otros significados para transversalizar lo simbólico, lo cotidiano, lo cultural, lo científico y lo vivencial.

El reto es consolidar una universidad más sensible, exhaustiva, distinguida por educar para la vida, integradora de la ciencia y las humanidades, impulsora de un saber autóctono y un pensamiento emancipador, formadora de ciudadanos dispuestos a relacionarse con su contexto de una forma creativa y desde una mirada ética, democrática y plural.

La transdisciplinariedad, como sustento de la universidad constituyente, enuncia una invitación para la comprensión y consolidación del acto formativo e investigativo como algo más humano, multidimensional, intercultural, reconocedor de la incertidumbre y la diversidad, y de un saber para el abordaje de problemas sociales desde un ejercicio 
de transformación permanente. Se trata, en otras palabras, de fomentar una actitud que ayude en la superación de las estructuras universitarias constituidas para desplazar la homogeneización y reemplazar la fragmentación con un principio de realidad que emerja de la coexistencia de una pluralidad compleja y una diversidad epistemológica.

Dados los planteamientos previamente señalados se presenta la siguiente interrogante: ¿qué premisas se han de considerar para migrar de la universidad constituida hacia la universidad constituyente? Entre las premisas o principios que podrían ser considerados para dinamizar tal migración se tienen:

Asumir la formación como un sistema en meta espiral de idas y venidas, esta variabilidad permitira hacer emerger y entrelazar los saberes, convirtiendo la incertidumbre en un elemento diferenciador y complejizador del proceso educativo.

Coadyuvar a los sujetos en la búsqueda de un equilibrio con el contexto en el que están inmersos, promoviendo la libertad de pensamiento, la reflexión, la crítica, la autocrítica, soportado en la noción de autonomía-dependencia.

Abordar los procesos de transformación cultural, educativa, política y económica que demanda la sociedad, resaltando lo investigativo y lo innovador.

Promover la investigación de los problemas u objetos de estudio desde múltiples miradas, favoreciendo una racionalidad dialógica, prospectiva y generadora de soluciones. (Acosta, 2016, p. 52)

La universidad constituyente, en consecuencia, no se asume como utopía o abstracción idealista, esta habita en los sujetos que la piensan y construyen a modo de reflexión desde la transdisciplinariedad, vista como "un diálogo entre formas" (Pérez, Alfonzo y Curcu, 2013, p. 25). En este sentido, las diferentes disciplinas y las diversas epistemes, los procesos de conceptualización y la actitud transdisciplinar son parte de ese diálogo, permitiendo fomentar la comprensión de la complejidad de las relaciones humanas, al promover la integración de los saberes.

Desde lo anterior, se asumiría al ser humano en relación con el conocimiento, consigo mismo y con los otros, para que conozca y esté consciente de cómo crearse y recrearse en todo momento. Así, la universidad constituyente animará a sus actores no para reproducir 
conocimientos sino para generar saberes para la vida. El colectivo universitario, en consecuencia, ha de estar consciente de que su quehacer estará rubricado por una faena en contraposición de los dispositivos, signos y códigos que inundan actualmente cada espacio de la sociedad moderna, por lo que en la medida que se integran más a su contexto territorial irán generando ámbitos que harán brotar sus propias prioridades, en los límites de novedades transitorias, que conllevan inevitablemente hacia la vertiginosa inmovilidad a la que se refiere Kurnitzky (1999, según se cita en Pereira, 2003).

La universidad constituyente, adicionalmente, coadyuvará a demoler los metarrelatos entendidos como "esos grandes proyectos, al tiempo reduccionistas y totalitarios, que se erigen con pretensiones de imponer valores, reglas y conductas en un mundo globalizado" (Guillaumin, 1996, según se cita en Hernández, Castañeda y Del Castillo, 2000 , p. 16). Estas formas de totalidad desechan "la pluralidad y diversidad de los tipos de saber, de los modos de realidad, de las formas de vida y de cultura" (Almarza, 2006, p. 37); en consecuencia, dicha universidad acogerá una formación y una investigación abierta a lo transcultural entendido como:

la apertura de todas las culturas a aquello que las atraviesa y las sobrepasa ... es ante todo una experiencia irreducible a toda teorización ... nos indica que ninguna cultura constituye un lugar privilegiado desde donde se puedan juzgar las demás culturas. (Nicolescu, 1996, p. 78)

Por otra parte, la formación y la investigación asumidas solo para el mercado globalizado deben ser reemplazadas por una concepción que dinamice la autoformación del sujeto y la formación ciudadana, esta última vista como la democratización de los espacios académico-comunitarios para "la recuperación de una política con la capacidad propia de los ciudadanos" (Fontalvo, 2008, p. 110). Los ciudadanos, vale decir, se definen por su solidaridad y responsabilidad en su ámbito local, regional, nacional, latinoamericano y planetario. Es la evolución y el fondeo de esta consciencia de pertenencia a la madre tierra lo que permitirá la germinación de un sentido de interconexión para sensibilizar los vínculos humanos, por tanto, la universidad "debe no sólo contribuir a una toma de conciencia de nuestra Tierra-Patria, sino también permitir 
que esta conciencia se traduzca en la voluntad de realizar la ciudadanía terrenal" (Morin, 1999, p. 4).

Con base en lo expuesto anteriormente, la universidad constituyente, desde la transdisciplinariedad, trabajará en favor de la "evolución de su misión, hoy en día un poco olvidada -el estudio de lo universal" (Nicolescu, 1996, p. 98). Esto con la intención de inyectar en el ámbito terrenal una cultura para coadyuvar a los ciudadanos a vivir sus destinos, defender y promover en el contexto social, educativo, cultural y político los valores intrínsecos del humanismo.

\section{Conclusiones}

La universidad constituida, en tanto instancia holográmica del entorno al que pertenece, apuesta por una disciplinariedad apegada a sus fundamentos epistemológicos, por lo que sus nodos de desarrollo amplían, y promulgan, la hegemónica racionalidad científica para la validación del conocimiento. Ello conlleva la cuantificación trivial de sus procesos, así como la descontextualización y fragmentación de los estados de conciencia epistémicos de los esquemas de conocimientos generados, lo que acarrea discontinuidades en sus procesos de formación e investigación que difícilmente la lógica disciplinar podrá contener.

En contraposición, la universidad constituyente, dado el papel que le corresponde protagonizar a la humanidad en el presente siglo, habrá de impulsar solidaridades y entendimientos basados en el respeto a la diversidad de pensamiento y el fomento de una conciencia ética, que promueva la construcción individual y colectiva del saber. Para alcanzar tan anhelado horizonte, ha de sustentarse en la transdisciplinariedad ya que a través de ella la relación dialógica-dialéctica, que se produce entre disciplinas, quedará implicada en la generación del saber y en la concepción del bucle formación-investigación como vínculo intersubjetivo, lo que entraña la posibilidad para fortalecer el diálogo de saberes. Así, los escenarios epistemológicos que surjan a lo interno de la universidad constituyente contribuirán con la fractura de los obstáculos que presentan tanto el conocimiento como la realidad.

Se despejarían, con dicha fractura, los escenarios para contribuir con un proceso pensado no solo para la formación cultural, sino para deconstruir y reconstruir una forma de intervención política que contribuya con la reforma del pensamiento. En consecuencia, dicha reforma 
ha de posibilitar que los sujetos puedan asumir y dar respuestas pertinentes a los desafíos que germinen en el presente siglo.

Cabe destacar que la universidad constituyente no se asume como homogeneizadora ni hegemonizadora, por el contrario, se concibe diversa, dinámica, compleja, multidimensional, pues se nutrirá con categorías y perspectivas que permiten pensarla desde la transdisciplinariedad. En otras palabras, despierta las utopías para construir puentes entre el conocimiento, el saber, la historia del hombre, del planeta, que acarreen el rescate del vitae, de la vida, requisito imprescindible para fortalecer el vínculo universidad-sociedad que conlleve hacia un nuevo tipo de humanismo.

\section{Referencias}

Acosta, J. (2016). Ejes de formación para los estudios avanzados: una propuesta para la Universidad Politécnica Territorial. Revista Conocimiento Libre y Licenciamiento, 14(7), 45-59.

Acosta, J. (2017). La epistemología del sur: una mirada desde el pensamiento de Boaventura de Sousa. Revista Cognosis, 2(2), 49-58.

Acosta, J. (2018). Hacia una nueva conciencia de la ciencia desde la realidad latinoamericana. Revista Pertinencia Académica, 6(1), $1-28$.

Almarza, F. (2006). Convergencia transdisciplinar: una nueva lógica de la realidad. Revista Tharsis, 7(2), 29-42.

Becerra, O. (Ed). (1996). Los efectos perniciosos de la racionalidad instrumental en el espacio escolar y la necesidad de recuperar la educación como el lugar para la confrontación y la búsqueda de nuevos horizontes. Caracas: Encrucijada Educativa V Congreso Internacional de Pedagogía Latinoamericana y del Caribe. Ediciones Los Heraldos Negros.

Becerra, O. (1997). El discurso y la racionalidad científica prevaleciente en nuestros ámbitos académicos universitarios. Revista Educación y Sociedad, 1(1), 60-74.

Castro, G. (1998). Hermenéutica y posmodernidad. Revista Apuntes Filosóficos, 3(13), 62-77.

Cubillán, J. (2008). Transescuela: el pensamiento de la complejidad pedagógica. Cumaná: Fondo Editorial UDO. 
De Sousa, B. (2008). La universidad en el siglo XXI: para una reforma democrática y emancipadora de la universidad. Caracas: Ediciones Centro Internacional Miranda.

De Sousa, B. (2010). Descolonizar el saber, reinventar el poder. Montevideo: Ediciones Trilce.

Fontalvo, R. (2008). Educar en la Complejidad. Caracas: Ediciones U.S.B.

Freire, P. (1980). La educación como práctica de la libertad. Ciudad de México: Siglo Veintiuno Editores.

González, J. (s. f.). Teoría educativa transcompleja: educación compleja y transdisciplinaria. La Paz: Ediciones Rizzo.

Hernández, L., Castañeda, E. y Del Castillo, A. (2000). La transdisciplinariedad, una acción prioritaria para la educación superior a comienzos del tercer milenio. Revista Universitaria Pedagógica, $5(1), 12-24$.

Lanz, C. (2007). A propósito del enfoque curricular que proponemos para la constituyente universitaria: los aportes de la teoría critica a la transformación de la educación superior. Recuperado de http://www.aporrea.org/educacion/a36793.html

Lanz, R. (2010). Diez preguntas sobre transdisciplinariedad. Revista AGORA, 1(16), 44-59.

Lanz, R. y Fergusson, A. (2005). La reforma universitaria en el contexto de la mundialización del conocimiento. Caracas: Ediciones del Observatorio Internacional de Reformas Universitarias.

Morin, E. (1992). El método III: el conocimiento del conocimiento. Madrid: Editorial Cátedra.

Morin, E. (1993). Tierra patria. Barcelona: Editorial Kairos.

Morin, E. (1997). Introducción al pensamiento complejo. Barcelona: Editorial Gedisa.

Morin, E. (1999). Los siete saberes necesarios para la educación del futuro. Madrid: Ediciones UNESCO.

Morin, E. (2002). La cabeza bien puesta. Buenos Aires: Editorial Nueva Visión.

Morles, V. (2004). (Ed). La universidad latinoamericana actual: necesidad de replantear su misión. Caracas: Editorial Metrópolis.

Nicolescu, B. (1996). La transdisciplinaria manifiesto. Ciudad de México: Edición 7 saberes. 
Pereira, L. (2003). Una apuesta estratégica en la educación: la concepción del ser en su unidad compleja. Revista UMBRAL 2000, 2(4), 22-38.

Pérez, E., Alfonzo, N y Curcu, A. (2013). Transdisciplinariedad y Educación. Cuadernos Educere, 17(56), 15-26.

Salazar, J. (2003). Convergencia e institucionalidad en la educación superior. Revista Calidad de la Educación, 19(2), 6-15.

Vilar, S. (1997). La nueva racionalidad, comprender la complejidad con métodos transdisciplinarios. Barcelona: Editorial Kaidós.

Zemelman, H. (1992). Los horizontes de la razón I: dialéctica y apropiación del presente. Ciudad de México: Editorial Anthropos. 
